\title{
O problema da causalidade em medicina
}

Vitor Rodrigues*

\section{RESUMO}

Em Saúde somos frequentemente chamados a fazer julgamentos sobre se uma exposição causa uma doença. Para podermos realizar julgamentos correctos é necessário que o raciocínio obedeça a critérios padronizados, sendo os mais frequentes os enunciados por Bradford Hill.

Adicionalmente, o desenho da investigação deverá a obedecer modelos diversos, com diferentes características arquitecturais e diferentes níveis de prova epidemiológica.

Outro factor extremamente importante é também o reconhecimento e a minimização de erros e desvios, particulares de cada tipo de estudo e gerais a toda a investigação epidemiológica.

Palavras-Chave: Causalidade; Tipos de Estudos; Vièses.

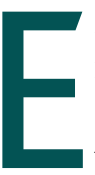
m Saúde somos frequentemente chamados a fazer julgamentos sobre se uma exposição causa uma doença. Mesmo em tempos antigos, navios que atracavam com marinheiros doentes eram colocados em quarentena, doentes com patologia infecciosa eram afastados das cidades, e até intervenções divinas ou hábitos «reprováveis» eram responsabilizados pelo aparecimento ou existência de doença.

Em meados do Século XIX, aquando do advento da «teoria dos germes», Henle e o seu pupilo Koch formularam hipóteses a partir dos quais se poderia fazer a inferência em como um determinado organismo vivo causaria uma doença particular. Na sua forma mais simples os postulados eram três:

- organismo encontra-se sempre quando há doença,

- organismo não é encontrado em nenhuma outra doença,

- organismo, isolado a partir de alguém com a doença e cultivado e desenvolvido através de várias gerações, produz a doença.

Quase um século depois, em 1964, quando vários estudos responsabilizaram o consumo de tabaco como causa de cancro do pulmão, houve uma enorme discussão sobre se tal raciocínio causal poderia ser aplicado às doenças crónicas. Para a ultrapassar, a mesma entidade e um artigo publicado por Sir Austin Bradford Hill

*Professor Associado da Faculdade de Medicina da Universidade de Coimbra em $1965^{1}$, avançaram critérios que poderiam ser usados para avaliar se uma associação poderia ser considerada como causal:

- Força: uma associação forte tem maior probabilidade de ser causal do que uma associação fraca, já que esta tem maior probabilidade de ser espúria, seja por viés, confundimento ou acaso. No entanto, uma associação fraca pode, apesar disso, ser causal.

- Consistência: se a associação se observa repetidamente em diferentes populações e em diferentes circunstâncias, tem maior probabilidade de ser causal do que se se tratar de uma observação isolada. No entanto, uma falta de consistência não afasta uma ligação causal; e pode também acontecer que uma causa apenas o seja na presença de factores adicionais e/ou concomitantes.

- Especificidade: o conceito aqui retratado implica que a causa apenas conduzirá a um efeito, não a múltiplos efeitos. Trata-se de um raciocínio mais adaptado à patologia infecciosa, sendo-o em manifestamente menor grau a outras patologias. Por exemplo, o consumo de tabaco é um factor de risco para diversas doenças.

- Temporalidade: a causa precede sempre o efeito. Não existe qualquer dúvida relativamente à validade deste critério.

- Gradiente biológico: o conceito tem subjacente a presença de um gradiente dose-resposta, isto é, se o 
aumento da exposição conduz a uma aumento do efeito, também aumenta a probabilidade de essa associação ser causal. No entanto, a não verificação desse gradiente não nega uma associação (ex. DES e adenocarcinoma da vagina, amianto e mesotelioma) e a sua existência não prova necessariamente causalidade (poderá até ser devido a viés ou confundimento).

- Plausabilidade: segundo o qual a ideia de causalidade tem de ser biologicamente plausível. No entanto, durante muitos anos não se «acreditou» que a úlcera gástrica ou o cancro do colo uterino poderiam ter uma componente infeccioso causal.

- Coerência: a assumpção de causalidade deverá estar ligada a outras observações (por exemplo, a relação causal entre consumo de tabaco e cancro do pulmão era coerente com as observações de que os fumadores tinham displasia do epitélio brônquico). No entanto, a ausência de coerência não afasta uma relação causal.

- Evidência experimental: os dados originados por experimentação animal ou humana ajudam a implicar a causalidade de uma exposição, sobretudo através do afastamento de factores como a subjectividade individual, o viés ou o confundimento.

- Analogia: se uma exposição está responsabilizada por uma doença, assume-se que outras exposições semelhantes poderão provocar doenças semelhantes (por exemplo, se a talidomida provoca malformações fetais, outros medicamentos administrados durante a gravidez poderão também provocar efeitos semelhantes). No entanto, é uma generalização demasiado ampla para, na maioria dos casos, ser útil. Embora se trate de uma abordagem muito útil numa avaliação geral do problema da causalidade, a realidade é bastante mais complexa do que emerge dos anteriores critérios. Bastará pensar no que conhecemos sobre factores de risco e na sua capacidade de actuação (aceleração ou travagem) na cascata epidemiológica da causalidade. E a sua classificação, por exemplo, em factores de risco necessários, suficientes, potenciadores, adjuvantes, desencadeantes, ajudará a compreender esta complexidade, que as metodologias e técnicas de investigação pretendem esclarecer e quantificar. ${ }^{2,3}$

Na realidade, o que normalmente queremos signifi- car por «causalidade» é o «aumento de probabilidade», e um dos problemas mais importantes reside na sua inferência da população para o indivíduo e do indivíduo para a população. ${ }^{2-4}$

\section{MODELOS DE ESTUDO, VALIDADE INTERNA E HIERARQUIZAÇÃO DE PROVA}

O modo de questionar as associações e relações de causalidade pode ser colocado de variadas maneiras. Conhecer as determinantes de uma doença, antecipar o prognóstico da doença, questionar a mais-valia terapêutica de um procedimento, identificar variáveis que influenciem a eficácia e a eficiência dos vários tratamentos disponíveis, associar prognóstico à acção terapêutica.

E essa avaliação (existência ou não de associação e relação causal) deve compreender, pelo menos, dois aspectos fundamentais: a identificação do efeito e a sua quantificação.

É no âmbito desta avaliação que devem ser compreendidos todos os modelos de estudo disponíveis em investigação em saúde,,$^{5-7}$ sendo a sua classificação «académica» e «pedagógica» uma actividade mental estimulante mas sempre adaptada aos dois objectivos atrás descritos (Figura 1).

A tentativa de responsabilização de uma exposição como factor de risco poderá, na sua forma mais simples, revestir o estudo de casos individuais ou de conjuntos de casos individuais. São estudos em que não existe qualquer grupo de controlo objectivo, embora possa ser realizada uma actividade mental de comparação (empírico) com a experiência ou conhecimento individuais do investigador.

Embora tais estudos possam ser passíveis de validação com recurso a, por exemplo, os critérios de Bradford-Hill, e que possam evidenciar existência (pelo menos aparente) de, por exemplo, temporalidade, coerência, plausabilidade biológica, consistência, a sua falta de «rigor científico» radica em características gerais como a não existência de grupo de controlo com quem contrastar e quantificar as diferenças observadas, de possibilidade de serem casos que não representam a maioria de indivíduos em circunstâncias análogas, de possibilidade de modificação de comportamentos devidos à atenção do investigador (o chamado «efeito Hawthorne») 


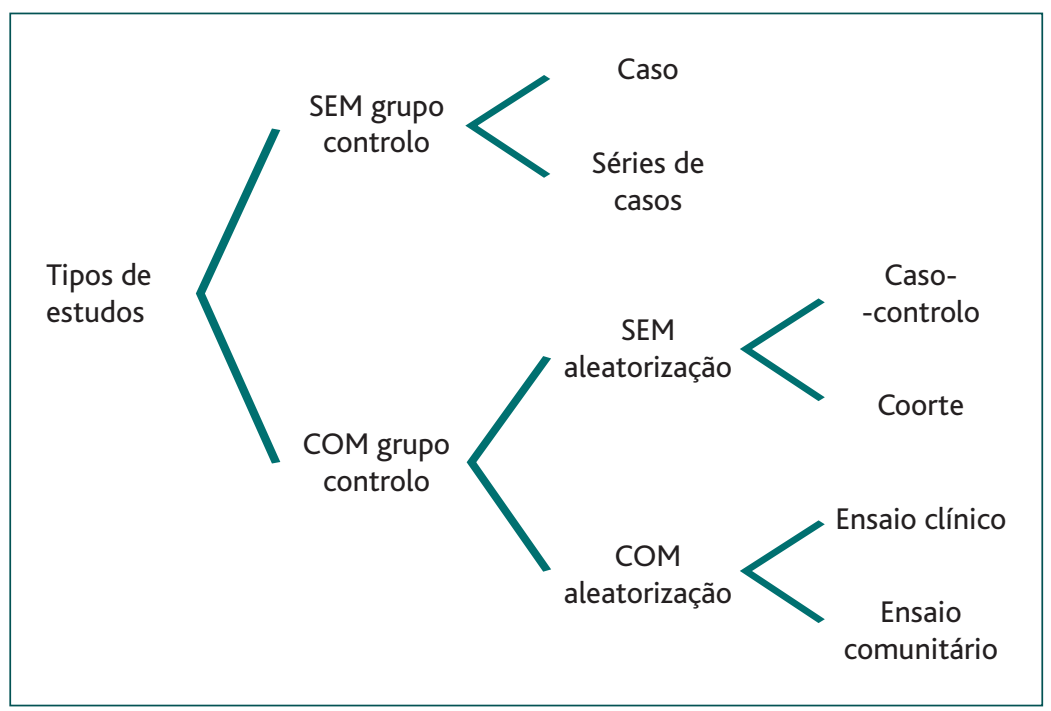

Figura 1. Estudos sem e com grupo de controlo. entre os efeitos detectados e as exposições consideradas, pelo que qualquer diferença na incidência poderá ser alocada ao efeito da exposição que diferencia as duas coortes.

Um estudo deste tipo tem a grande vantagem de acompanhar (com supervisão) os acontecimentos que vão surgindo por acção (positiva ou negativa) da presença ou ausência de exposição, além de que vão permitir a incidência $\mathrm{da}(\mathrm{s})$ doença(s) relacionadas nos grupos em estudos. No entanto, o seu desenho implica que sejam mais caros, seja em termos de recursos financeiros como de pessoal) e que necessitem de grandes efectivos populacionais, sobretudo se o efeito é de baixa incidência, e

\section{Estudos com grupo de controlo}

Os estudos com grupo de controlo permitem uma comparação directa e objectiva entre o grupo em estudo e um outro grupo que não possuirá as mesmas características em apreciação; esta distinção é fundamental para a detecção e quantificação das variáveis em estudo, sendo a sua validade tanto maior quanto mais potente for o controlo (e afastamento) dos viéses ${ }^{8}$ que poderão distorcer a eventual ausência ou presença de relação causal.

Estes estudos podem ser de dois tipos fundamentais: aqueles em que a variável em estudo é introduzida (idealmente de forma aleatória) pelo investigador («experimentais» - como exemplo temos os ensaios clínicos e os ensaios de intervenção comunitária) e aqueles em que aquele se limita a observar a evolução da variável em estudo («de observação»-como exemplo temos os estudos de metodologia de coorte e os estudos de metodologia caso-controlo). A sua diferença fundamental consiste no ponto de partida da «história natural da doença» ou da relação de causalidade («a causa precede sempre o efeito").

Os estudos de metodologia de coorte (estudos prospectivos) partem da identificação da presença ou da ausência de exposição ao factor de risco em causa (e cada uma destas duas populações chama-se uma «coorte»). A identificação do aparecimento ou não do efeito vai permitir que seja possível avaliar a relação que, tradicionalmente, obriguem a um seguimento temporalmente dilatado, sobretudo se são realizados para investigar causalidades com grande tempo de latência.

Os estudos de metodologia caso-controlo (estudo retrospectivo) fazem uma abordagem oposta, isto é, parte da identificação do efeito (normalmente, doente ou não doente) para pesquisar se o grupo de casos e o grupo de controlos estiveram ou não expostos à(s) exposição(ões) de interesse.

Os estudos deste tipo são particularmente indicados para se estudar a etiologia de acontecimentos pouco frequentes ou raros. São também mais rápidos de executar (não é necessário esperar pelo aparecimento do efeito), necessitam de efectivos populacionais mais reduzidos, sendo, deste modo, mais adequados numa perspectiva de maior facilidade e rapidez de concretização.

\section{Viéses mais frequentes ${ }^{8}$}

No caso dos estudos de metodologia de coorte identificam-se quatro tipos principais de desvios: selecção, migração, observação e por presença de factores de confusão.

No primeiro caso, trata-se do erro sistemático que determina que as coortes não sejam comparáveis em todas as variáveis prognósticas, devendo-se à natureza observacional do estudo; na realidade, se o investigador não tem possibilidade de alocar (por randomização, idealmente) os participantes às exposições em estudo. 
O viés de migração refere-se ao abandono, por várias razões, da coorte (em que se iniciaram) por alguns dos indivíduos em estudo; tem a desvantagem de diminuir o efectivo inicialmente calculado, bem como poder acontecer que esse conjunto de indivíduos ter características em comum, podendo ele mesmo ser uma «coorte de abandono por característica comum».

O viés de observação refere-se sobretudo ao facto do investigador poder incluir, consciente ou inconscientemente, nas suas observações aspectos de valorização pessoal de factores em estudo.

Por fim, o viés por presença de factores de confusão aparece quando uma variável que não está incluída, pelo menos directamente, na cascata de causalidade, se encontra relacionada na associação entre os eventos (exposição e efeito) em estudo. Esta associação confundente obriga a cuidados especiais no desenho e na avaliação do estudo.

No caso dos estudos de metodologia caso-controlo identificam-se 3 tipos principais de desvios: selecção, informação (ou memória) e por presença de factores de confusão.

No primeiro caso, este viés produz-se quando os casos e os controlos são seleccionados de maneira sistematicamente diferente em relação com a possibilidade de que tenham estado ou não expostos ao(s) factor(es) de risco de interesse. Deve haver o cuidado de que os casos sejam representativos da generalidade dos casos e que estejam num momento representativo da história natural da doença em causa. Por outro lado, os controlos devem ser seleccionados de tal modo que sejam, o mais possível, semelhantes aos casos no que diz respeito às variáveis em estudo (apenas diferirem na doença em estudo).

O segundo viés refere-se ao facto da informação a pesquisar estar naturalmente mais presente e correcta nos casos do que nos controlos (aqueles estarão mais sensíveis à possibilidade de existência dos factores de risco a estudar); mas também o entrevistador poderá ser mais insistente relativamente à informação que ela pensa ser mais importante.

O terceiro viés tem as características gerais semelhantes ao atrás descrito.

\section{Estudos com grupo de controlo aleatorizados}

Nestes tipos de estudos (ensaio clínico e ensaio de in- tervenção comunitária) existe um factor fundamental: a colocação do factor diferenciador em estudo é realizada pelo investigador. $E$ a diferença que existe entre os dois tipos principais radica na unidade de análise: o indivíduo no primeiro caso e o grupo no segundo caso. Deve notar-se que estes estudos são extensões, metodologicamente mais apuradas, dos estudos de metodologia de coorte atrás descritos.

A sua metodologia geral consiste na selecção de uma amostra a partir de uma população (através da aplicação de critérios de inclusão e de exclusão rígidos), sendo depois aquela distribuída pelos grupos (mínimo de dois) adequados.

Possui características fundamentais como sejam a cuidada selecção dos participantes, o tamanho da amostra, a distribuição por grupos aleatória, a possibilidade de cruzamento de exposições e a ocultação.

Os participantes são grupos altamente homogéneos (e, idealmente, representativos) com controlo estrito das variáveis que podem mascarar a associação causal, o que permite uma maior certeza na avaliação da validade externa do estudo. Para tal, utilizam-se critérios de inclusão e de exclusão bem definidos (embora mais ou menos restritivos), o que permite uma maior representatividade para grupos externos que partilham a mesma situação.

Por outro lado, a selecção e quantificação cuidadas da amostra, através de factores como a homogeneidade dos participantes, a definição estrita sobre as diferenças que se desejam detectar e os erros tipo I (probabilidade de rejeitar a hipótese nula quando esta é verdadeira) e tipo II (probabilidade de confirmar a hipótese nula quando esta é falsa) que se consideram aceitáveis. São factores que irão influenciar decisivamente o quantitativo amostral a incluir.

A sua distribuição aleatória por grupos (a grande diferença relativamente ao estudo de metodologia de coorte) permite que aumente a probabilidade da comparabilidade inter-grupos e de representatividade da investigação. Também evita distribuições enviesadas por parte do investigador e de cada indivíduo incluído no estudo.

É possível, num estudo desta natureza, cruzar exposições ao longo do estudo. Para além do benefício ético que consiste na (re)colocação do indivíduo na exposição (terapêutica) que se verifica ser mais adequada 
para si (o que pode implicar a sua saída do ensaio), a sua grande vantagem reside na concomitante redução do número de indivíduos necessários para a investigação e na possibilidade de avaliação de mais do que um efeito.

A ocultação será também um factor fundamental; ela permite afastar o factor subjectivo individual do investigador no tratamento e monitorização dos expostos (ocultação simples), no afastamento do «efeito placebo» no indivíduo exposto (ocultação dupla) e na garantia de monitorização e avaliação não enviesadas.

\section{Indicadores gerais de resultado}

O facto dos estudos atrás apresentados pesquisarem, na sua forma mais simples, uma associação entre duas variáveis dicotómicas permite que a exposição e o efeito possa ser colocado numa simples tabela de $2 \times 2$ (Quadro I).

\begin{tabular}{|c|c|c|c|}
\hline \multicolumn{4}{|c|}{$\begin{array}{l}\text { QUADRO I. Tabela binária para cálculo da associação } \\
\text { entre exposição e efeito }\end{array}$} \\
\hline & Efeito + & Efeito - & Total \\
\hline Exposição + & A & $b$ & $a+b$ \\
\hline Exposição - & $\mathrm{C}$ & $d$ & $c+d$ \\
\hline Total & $a+c$ & $b+d$ & \\
\hline
\end{tabular}

No estudo de metodologia de coorte (ou ensaio clínico) a incidência entre os indivíduos expostos é de $a /(a+b)$; a incidência entre os não expostos é de $c /(c+d)$; o risco relativo é de $(a /(a+b)) /(c /(c+d))$. Tal indicador denomina-se por «risco atribuível» (à exposição).

No estudo de metodologia caso-controlo, uma vez que não é possível termos taxas de incidência (lembre-se que partimos da doença e não da exposição), há necessidade de recorrer a uma estimativa do risco relativo (odds ratio- OR) que se calcula por $a^{*} c / b^{*} d$.

É habitual considerarmos o risco relativo como um risco «individual» (próprio de indivíduos sujeitos a um factor de risco) o risco atribuível como um risco «colectivo» (próprio de populações sujeitos a um factor de risco).

A anterior explanação geral das metodologias disponíveis e dos viéses passíveis de acontecer permitem que se possa realizar uma hierarquização da robustez da prova (Figura 2).

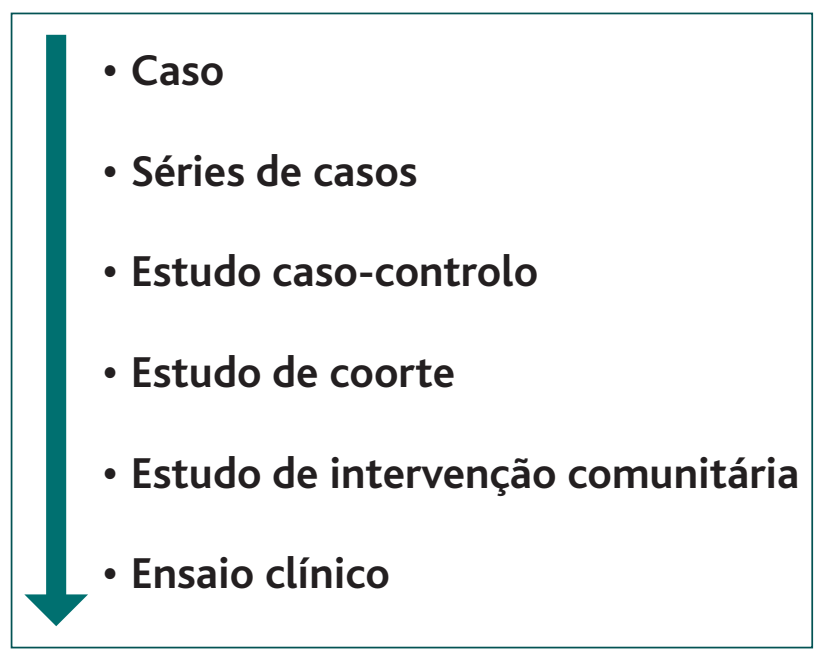

Figura 2. Hierarquização da robustez da prova.

\section{REFERÊNCIAS BIBLIOGRÁFICAS}

1. Hill $A B$. The environment and disease: association or causation? Proc Royal Soc Med 1965 May; 58: 295-300.

2. Weed DL. Causal criterian and Popperian refutation. In: Rothman KJ, editor. Causal Inference. Chestnut Hill: Epidemiologic Resources; 1988. p. 15-32.

3. Susser M. Falsification, verification and causal inference in epidemiology: reconsiderations in the light of Sir Karl Popper's philosophy. In: Rothman KJ, editor. Causal Inference. Chestnut Hill: Epidemiologic Resources; 1988. p. 33-58.

4. Pearl J. Causality: models, reasoning and inference. Cambridge: Cambridge University Press; 2000.

5. Rothman KJ. Principles of epidemiology. Oxford: Oxford University Press; 2002

6. Silman AJ, MacFarlane GJ. Epidemiological studies: a practical guide. Cambridge: Cambridge University Press; 2008.

7. Strom BL. Study designs available for pharmacoepidemiology studies. In: Strom BL, editor. Pharmacoepidemiology. 2nd ed. Chichester: John Wiley and Sons; 2000. p. 247-62.

8. Sackett DL. Bias in analytic research. J Chronic Dis 1979; 32 (1-2): 51 $-63$.

\section{ENDEREÇO PARA CORRESPONDÊNCIA}

Vítor Rodrigues

E-mail:vrodrigues@netcabo.pt 


\section{RESUMO}

In Health, very often we need to judge if an exposure is a cause of a disease. In order to perform correct judgements, reasoning must obey to standardized criteria, being those published by Bradford Hill the most commonly used.

Furthermore, research architecture must comply with different models, with different design characteristics and different levels of epidemiological proof.

Another very important aspect is the recognition and minimization of errors and bias, some particular to each type of study and other general to the epidemiological research.

Keywords: Causation; Types of Studies; Bias. 